\title{
Nanoscale Particles in Medical
}

\section{Products}

\author{
By Hermann Nirschl*
}

Composite materials are used as an alternative for amalgam fillings or as fixing materials in dentistry. Similar types of materials can be used as bone replacement materials such as replacements for the small bones

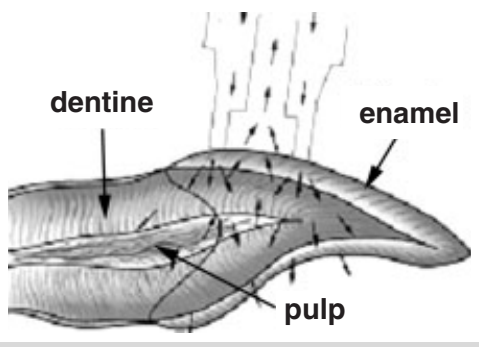
in the ear. Materials of this type possess a high proportion of filler which can only be achieved by specific design of the filler matrix.

\section{Introduction}

The following describes the application of nanoscalic fillers in medical products which are in use in dental practise. Composite materials are used as an alternative for amalgam fillings or as fixing materials in dentistry. Similar types of materials can be used as bone replacement materials such as replacements for the small bones in the ear. Materials of this type possess a high proportion of filler which can only be achieved by specific design of the filler matrix. The filler material is embedded in an acrylate monomer which is hardened to a polymer matrix by a light initiated reaction. The pasty end product, which can have a viscosity up to 50,000 Pas., is used in clinical practise with specially adapted preparation techniques.

\section{Preparation of the Filler}

The property profile of these densely packed composite systems is equally dependent on the physical chemical properties of the pure matrix and on the properties of the filler. In addition to the physical-chemical surface properties of the filler particles in this two phase system, the packing and permeability of the filler are also of particular significance and can influence quite significantly the mechanical properties, aesthetics, shrinkage and also the ease of handling of the composite. The targeted implementation of the desired property profile requires the use of very fine custom made particles of the nanoscale size range and a correspondingly adapted technique for the preparation of the powder.

The requirements of the filler can be described in the sense of structure-property relationships. The high strength of the prepared composite materials is dependent on the break strength of the filler. The strength of the filler is a key require- ment for its application. The resistance to abrasion of the polymer during chewing in the mouth of the patient is also significant. The colour and ease of polishing are criteria which can only be realised with nanoscale fillers which provide a close approximation to the natural tooth structure. In the following text these criteria will be paid attention to. In addition the natural appearance and the biological suitability are also important.

It is known that, because of their large specific surface area and their physical properties, small particles sometimes show new and surprising properties. It is expected that the use of such particles will improve the strength, optical and rheological properties of materials and new properties can be expected as yet unknown of these high density systems. For a relatively low percentage of such a filler, a significant change in the characteristics of the material can be expected. It can also be expected that from the fineness of the particles or by a deliberately defined size distribution the degree of filling in these systems can be raised without negatively influencing the fluid properties of the materials. In this way shrinkage which can have a negative influence on the lifetime of composite materials can also be reduced.

There are basically three processes for the preparation of filler materials based on inorganic materials available.

\subsection{Preparation of the Filler by Milling}

The starting material must be ground to a nanoscale filler by a suitable technological process. It can be ground in a wet

[*] Prof. Dr.-Ing. Hermann Nirschl Universität Karlsruhe (TH) Institut für Mechanische Verfahrenstechnik und Mechanik Kaiserstr. 12, D-76128 Karlsruhe (Germany) 
state in an agitator bead mill. Bead mills belong to a group of grinding machines with lose grinding media. During operation the chamber volume is up to $85 \%$ filled with grinding beads. These grinding beads, usually steel, glass or ceramic are intensively moved by the agitator. The abrasiveness of the raw material must be taken into account otherwise unwanted colour changes can occur. The remaining free volume of the grinding chamber is filled with a suspension of a fluid (e.g. water, alcohol, oil) and the substance to be ground. In continuous operation the suspension is pushed through the chamber. The grinding beads are contained within the grinding chamber by the help of a separator. Rotating slits, sieves and recently also centrifuges are used as separators. The most important operating parameters (agitator speed, filling ratio of grinding bodies, properties of grinding bodies, volume flow and solid concentration of the suspension) can be freely chosen and so adapted to the required grinding task.

The increasing influence of particle-particle interaction in suspensions when average particle sizes are less than $1 \mu \mathrm{m}$ leads to agglomeration between the individual primary particles. The interactions are not only determined by the operating parameters but also by the surface properties of the substance to be ground. To ensure sizes of less than $1 \mu \mathrm{m}$ the single particles must be stabilised. Electrostatic stabilisation occurs by adsorption/desorption of surface specific materials and ions (co-ions) when particles are suspended in electrolyte solution, such stabilisation can be relatively easily attained by changing the $\mathrm{pH}$ value of the suspension. Figure 1 shows the results for three repeated attempts at grinding the same raw material. In this case the grinding limit is at approximately $500 \mathrm{~nm}$. This value can be further reduced with the help of additional stabilisation measures. ${ }^{[1]}$

\subsection{Synthesis of the Filler Material by Wet Chemical Methods or From the Gas Phase}

The synthesis of very fine particles can be achieved in two different ways. There are wet chemical methods in which particles are formed by precipitation or crystallisation. There are a variety of dry processes for the production of very fine and in particular nanoscale powders. The dry processes differ according to the phase of the starting material. The most common is the gas phase synthesis in which gaseous precursors are used. The gas phase process has the advantage over the wet chemical methods that the product powders are dry in the final stage and of a higher degree of purity. ${ }^{[2]}$

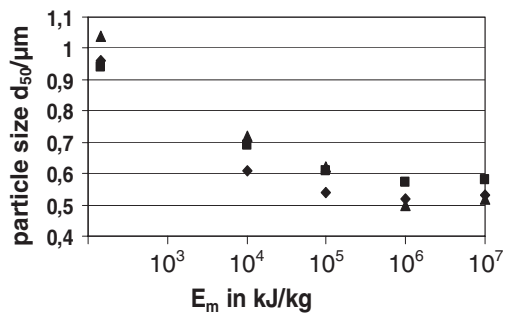

Fig. 1. Dependence of particle size on specific energy applied.
Current knowledge indicates that the chemical reaction and the nucleation process follow several formation mechanisms such as coagulation, surface growth or coalescence. Coagulation describes particle growth by collision with already available molecule clusters or particles and leads to larger and irregular aggregates. Particle growth also occurs by chemical reaction of the precursors on the particle surface whereby the disperse surface can also have a catalytic effect on the reaction of the precursors.

It is understandable that according to the dominance of the formation methods particle systems with completely different properties can arise. Such powders of very high quality have recently become commercially available. Additional processing steps which are adapted to the monomer used are required.

\subsection{Preparation of the Filler via Sol-Gel Process}

Silicic acid, many oxides, metals or proteins can form colloidal solutions. The particles suspended in the fluid are so small that they appear to form a single phase even though they do not interact in a molecular way with it as in a real solution. One speaks of a colloidal solution or a sol. The diameter of these colloidal particles is within the range 1-100 nm. The systems are mid way between real solutions and coarse disperse systems and so correspondingly colloidal solutions can be prepared either by particle size reduction of larger particles or by particle size enlargement of molecularly dissolved materials.

If a colloid which is stabilised by an electrical charge needs to be flocculated the charge on the colloid particles must be negated. A common way is to add particularly well absorbing counter charged ions. Generally solutions of hydrophobic colloids are very sensitive to the addition of an opposing electrolyte.

Nanomers (single primary particles in the nanometer scale) and nanoclusters (aggregates of primary particles) can be prepared by a sol-gel reaction. (see fig. 2). A sol of defined nanoscale particles is precipitated (e.g. by the addition of an acid) to form a gel and is dried under conditions which have been designed to avoid the formation of an agglomerate

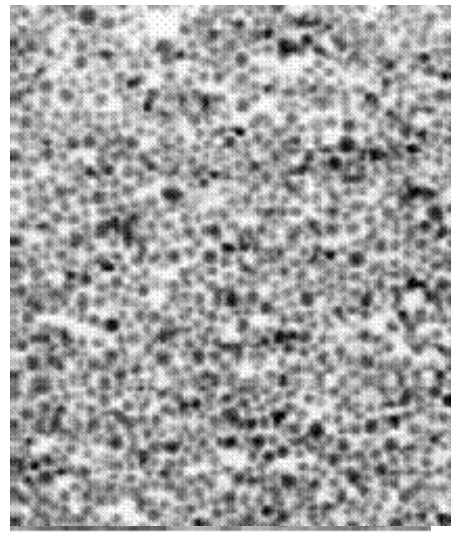

Fig. 2. REM-images of nanomers and nanoclusters /3/. 
which cannot be redispersed in a later stage. The trick is to obtain nanomers which can be redispersed in monomers of their primary particle size. An homogenous distribution of nanomers in monomers appears transparent.

Nanoclusters will be made from nanomers. Both fillers will by initially functionalised i.e. the surface structure will be changed to ensure a perfect dispersion of the monomer and that later binding on the polymer is successful. Simultaneously a nanostructure is made, i.e. the particle size distribution is modified, such that the natural colour of the tooth can be imitated. Converse to the so called "microfills" where the filler consists fundamentally of particles in the micrometer scale, the use of nanomers and nanoclusters can achieve a natural and accurate imitation of tooth colour.

A skilful combination of nanomers and nanoclusters can result in a higher degree of filling because an optimal particle size distribution can be found for the simultaneous optimisation of optical, mechanical and rheological properties of the monomer mixtures.

The natural structure of the tooth and its colour appearance

In order to understand the reasons for the effectiveness of nanoscale particles in composites for use in dentistry it is necessary to describe the anatomy of the tooth.

A tooth consists of an outer layer of enamel. Chemically this is calcium phosphate, which has a high mechanical hardness. Under this layer is the dentine which consists of crystals of calcium phosphate and collagen fibres. In the middle of the dentine is the pulp which contains nerves and blood vessels which supply the enamel and dentine with nutrients. (see Fig. 3).

The tooth colour of an adult is determined mainly by the dentine. The enamel plays a less important role in determining the colour. The most intense colours are in the region of the gums and are due to the thickness of the dentine and the thin layer of enamel. At the tip or crown of the tooth the influence of the dentine on the appearance is much less and the translucence of the enamel is more important. The appearance in the areas where dentine is more of an influence is more opaque than at the tip.

The natural appearance of the tooth is a result of many interactions between the tooth components and the mouth. If teeth are restored using exclusively composite materials with filler sizes in the micrometer scale the natural appearance cannot be retained because the fillers have no translucent component and so only reflect light. Light cannot shine through artificial structures or fillings. With metal crowns it is obvious. Enormous effort is required to reproduce the natural coloration. There is a material which can be used to restore a tooth which by the use of nanoscale particles appears translucent and so is much nearer to the sheen of a natural tooth. A best combination of nanoscale particles (nanomers and nano cluster) needs to be found which with the help of colour pigments can reproduce the colour and also the opalescent and translucent appearance of the tooth.

\subsection{Polishing}

The electron micrograph images (Fig. 4) show polished surfaces of composite materials. (i) The filler is in the micrometer scale. In polishing the high speed of the tool removes particles from the structure. Craters are left behind which make the surface appear rough and therefore unnatural. The use of fillers with a particle size distribution with sizes significantly less than $1 \mu \mathrm{m}$ can be better polished (microfills) (ii). An abrasion will not only remove the larger filler particles but also the finer ones. The polish and imitation of the natural sheen is significantly improved in comparison with the previous case. In the nanomer/nanocluster material (iii) the nanoclusters are only loosely attached to one another, so in the polishing process they are not torn from the structure as clusters but as single nanomers. This considerably raises the ease of polishing compared to conventional materials. The combination of nanomer/nanocluster also allows the gaps between the nanoclusters to be filled and so raises the degree of filling and the strength that can be reached.

\section{Redispersion}

Good redispersion is an indispensable requirement for the preparation of densely packed nanoscale composite materials. For technical reasons the powders cannot be prepared in their later matrix. The current state of knowledge is that fine highly aggregated particles have bad dispersive properties. The desired effects can only be developed in the powder if a most fine and even dispersion is achieved. Attempts are being made to achieve the required conditioning in-situ during the preparation. It is of particular importance that throughout the whole process the most modern particle measurement techniques and in-line quality control are employed



Fig. 3. Diagram of a natural tooth.
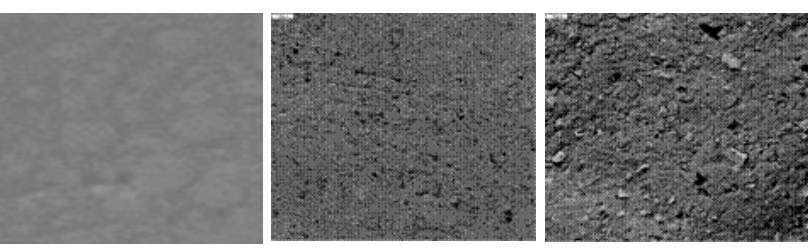

Fig. 4. (i) $\mu m$-Filler, (ii) Microfill, (iii) Nanomer/Nanocluster. 
in order to ensure the desired multifunctional powder properties, with respect to the possibilities of influencing the physical and chemical properties of the composite material.

\section{Summary}

The above text describes the use of nanoscale particles in composite materials used in dentistry. Methods of preparation of functionalised particles are explained and their application in the dental matrix. With the help of nanoscale particles new materials with previously unknown properties can be developed for medicinal products for production on the industrial scale however new and innovative processes are required.

Received: June 06, 2003

[1] F. Stenger, W. Peukert, S. Mende, J. Schwedes, Chem. Ing. Tech. 2002, 74, 994.

[2] T. Kodas, M. Hampden-Smith, Aerosol Processing of Materials, Wiley-VCH, Weinheim 1999.

[3] Technical Product Profile, Filtek ${ }^{\mathrm{TM}}$ Supreme, 3M ESPE, 2002. 